\title{
El material audiovisual en la enseñanza de la asignatura historia del derecho mexicano: de la reflexión crítica a la búsqueda de alternativas de difusión de la investigación histórico-jurídica
}

\author{
Audiovisual material in the teaching of Mexican legal history: from critical \\ reflection to the search for alternatives for the dissemination of historical-legal \\ research.
}

DOI: $10.46932 / \mathrm{sfjdv} 2 \mathrm{n} 2-162$

Received in: March 1st, 2021

Accepted in: May 30th, 2021

\section{Dra. Yvonne Georgina Tovar Silva}

Doctora, Maestra y Licenciada en Derecho por la Facultad de Derecho de la Universidad Nacional Autónoma de México. Magíster en Derecho Internacional: Comercio, Inversiones y Arbitraje otorgado por las Universidades de Chile y Heidelberg

Correo: ygtovars@derecho.unam.mx

\section{RESUMEN}

El presente documento pretende explorar las aportaciones del empleo del material audiovisual como una herramienta didáctica susceptible de sensibilizar al estudiante de la complejidad política, económica, social y cultural presente en la formación del sistema jurídico mexicano desde la época prehispánica hasta el siglo XXI. Dicha sensibilización es relevante para enriquecer el diálogo interdisciplinario, así como favorecer la comunicación de los resultados de la investigación histórico-jurídica a través de las tecnologías de información y comunicación. Bajo este esquema, se pretende reflexionar en torno al uso de las tecnologías de la información como parte de las técnicas de enseñanza del Derecho y los cambios que dichas tecnologías generan en el proceso de enseñanza-aprendizaje.

Palabras clave: Tecnologías de la Información y Comunicación, material audiovisual, técnicas de enseñanza del Derecho, investigación histórico-jurídica.

\begin{abstract}
This paper intends to explore the contributions of the use of audiovisual material as a didactic tool capable of sensitizing the student to the political, economic, social and cultural complexity present in the formation of the Mexican legal system from pre-Hispanic times to the 21st century. Such sensitization is relevant to enrich the interdisciplinary dialogue, as well as to favor the communication of the results of historicallegal research through information and communication technologies. Under this scheme, we intend to reflect on the use of information technologies as part of the teaching techniques of law and the changes that such technologies generate in the teaching-learning process.
\end{abstract}

Key words: Information and Communication Technologies, audiovisual material, law teaching techniques, historical-legal research. 


\section{INTRODUCCIÓN}

El objetivo del presente trabajo es explorar la manera en que el material audiovisual en la enseñanza de la asignatura Historia del Derecho Mexicano puede ser significativo para fomentar la reflexión crítica, enriquecer el diálogo interdisciplinario, así como explorar otras formas de exponer los resultados de las investigaciones relacionados con la conformación, evolución y elementos formativos del Derecho Mexicano. Dicha temática se enmarca en las reflexiones en torno al uso de las Tecnologías de la Información y Comunicación en la enseñanza de la asignatura Historia del Derecho Mexicano a nivel Licenciatura, así como los cambios esperados en la formación de los estudiantes.

Se estima que el empleo de los materiales audiovisuales elaborados por instituciones académicas representan una herramienta auxiliar para reforzar la necesidad de que el estudiante vincule el análisis del fenómeno jurídico con otras disciplinas, como la Historia o la Política, por ejemplo, que le permita recrear, y en su caso, cuestionar el contexto dentro del cual se emiten las normas jurídicas. De esta manera se busca que los estudiantes se sensibilicen de que el desarrollo del Derecho guarda una estrecha vinculación con la complejidad política, económica, social y cultural existente en un momento determinado. Asimismo, se busca exhortar a los estudiantes a identificar nuevas vías para difundir la investigación histórico-jurídica, susceptible de ser comprendida tanto por juristas como por el público en general. Por lo anterior, es conveniente continuar con la reflexión en torno a las técnicas de enseñanza del Derecho en la era digital, a efecto de identificar las ventajas que se pueden obtener de las tecnologías de la información y comunicación en el proceso de enseñanza-aprendizaje.

En este contexto, un primer tema a abordar explora brevemente los objetivos de la asignatura Historia del Derecho Mexicano, correspondiente al primer semestre, contemplada en el Plan de Estudios de la Facultad de Derecho de la Universidad Nacional Autónoma de México para contextualizar con un ejemplo la ubicación de dicha asignatura en la formación del jurista. En un segundo tema, se abordará la manera en que a través de las técnicas de enseñanza del Derecho se puede incorporar el empleo de los materiales audiovisuales y su relevancia en el proceso de enseñanza-aprendizaje. Finalmente, en el tercer tema se indagarán las aportaciones del material audiovisual en la asignatura Historia del Derecho Mexicano para fomentar la reflexión crítica del estudiante, enriquecer el diálogo interdisciplinario y favorecer nuevos enfoques de difusión de la investigación jurídica, lo que apunta a la relevancia de capacitar a los docentes para el adecuado uso de las Tecnologías de la Información y Comunicación.

Bajo este panorama, se continúa en la búsqueda de herramientas docentes que le permitan al futuro jurista contar con una sólida formación, apertura al diálogo e interés por la investigación jurídica, que en conjunto contribuyan a transformar la realidad social a través del Derecho. 
El presente trabajo se basa significativamente en una colaboración previa presentada en el marco del Quinto Encuentro Universitario de Mejores Prácticas de uso de Tecnologías de la Información y Comunicación en la educación realizado en la Universidad Nacional Autónoma de México.

2 GENERALIDADES EN EL ESTUDIO DE LA ASIGNATURA HISTORIA DEL DERECHO MEXICANO: UNA PERSPECTIVA A PARTIR DE LA ASIGNATURA DE

\section{LA FACULTAD DE DERECHO DE LA UNIVERSIDAD NACIONAL AUTÓNOMA DE MÉXICO}

Con la finalidad de contextualizar los alcances del empleo del material audiovisual en la enseñanza de la asignatura Historia del Derecho Mexicano, se explorarán los objetivos de dicha asignatura en la formación del jurista, en los términos contemplada en el Plan de Estudios 2019 de la Facultad de Derecho de la Universidad Nacional Autónoma de México ${ }^{1}$.

En el referido Plan se prevé que la asignatura Historia del Derecho Mexicano "proporciona conocimientos sobre las instituciones que antecedieron al orden jurídico mexicano contemporáneo, comparando con otros sistemas jurídicos del mundo y proporcionando las técnicas propias del análisis científico de las fuentes históricas del Derecho." (p. 86). Bajo este contexto, en el Plan de estudios en comento, se establece como objetivo de la asignatura la distinción de los antecedentes del Derecho Mexicano en las culturas de los pueblos originarios y el castellano y su impacto en la formación del Derecho Novohispano, así como la aparición y desarrollo del Derecho Mexicano desde la Independencia hasta el siglo XXI. Con el estudio de dicha asignatura se prevé adquirir ciertas competencias jurídicas referentes al examen de las instituciones jurídicas y los fenómenos políticos, sociales, económicos que contextualizaron al Derecho Prehispánico y Castellano que además influyeron en la configuración del Derecho Novohispano y del posterior Derecho Mexicano desde la Independencia hasta la actualidad, con el respectivo desarrollo de habilidades de investigación de gabinete y campo, así como destreza en la identificación de la naturaleza jurídica histórica de las leyes y de las instituciones, reforzando la identidad nacional al tiempo de apreciar la multiculturalidad de México y el mundo.

En este marco, la asignatura en comento junto con proporcionar conocimientos en torno a la formación del sistema jurídico mexicano, también contribuye a desarrollar competencias como la investigación y el diálogo que son significativas en el actuar del jurista.

\footnotetext{
${ }^{1}$ La referencia al Plan de Estudios 2019 de la Facultad de Derecho de la Universidad Nacional Autónoma de México, solamente se realiza con la finalidad de ubicar la asignatura de Historia del Derecho Mexicano en la formación del jurista. Excede de los alcances del presente trabajo emitir otro tipo de valoraciones, planteamientos o cuestionamientos al Plan de Estudios en comento.
} 
Para Pérez de los Reyes (2008), las Historia del Derecho Mexicano proporciona al estudiante beneficios como enriquecer su cultura jurídica, proporcionar los antecedentes histórico-jurídicos de las normas e instituciones que configuraron el actual sistema jurídico mexicano, contribuye a formar un criterio jurídico en la creación y aplicación del Derecho, así como generar una vinculación histórica de las especialidades del Derecho. En el mismo orden de ideas, Soberanes Fernández (2008) señala que la disciplina de la historia del Derecho permite comprender el Derecho en su totalidad, al fincar los conocimientos de una interpretación global del Derecho, al permitir tomar conciencia del momento histórico del cual son protagonistas. Por su parte, Margadant (2000) indica que las nociones históricas con que cuente el jurista le permitirán preguntarse acerca de las constelaciones de intereses sociales que ha dado lugar a la norma, la manera en que en su caso la norma jurídica frenó o estimuló ciertos desenvolvimientos sociales o políticos, las razones subyacentes en la transformación o eliminación de la norma, así como la debida interpretación de la norma.

Bajo este esquema, el análisis de la formación y evolución del sistema jurídico mexicano, contribuye a que el estudiante adquiera un sentido crítico bajo el cual sea posible comprender la manera en que las ideas y circunstancias políticas, económicas, sociales y culturales existentes en un momento dado inciden en el Derecho.

Pese a la relevancia formativa del estudio de la disciplina en comento, se ha apreciado un desinterés en incorporar dicha disciplina a los Planes de Estudio de la Licenciatura en Derecho en algunas instituciones de educación superior en México, a lo cual habría de sumarse que ha sido relativamente pocas las tesis que tienen como eje temático la Historia del Derecho Mexicano. En este marco, resulta conveniente identificar propuestas adicionales bajo las cuales es posible motivar el estudio de la Historia del Derecho Mexicano, que comprendería desde la reconsideración de los objetivos de la formación del jurista y la trascendencia que la Historia tiene en esa formación sobre todo para incorporar dicha asignatura en los Planes de Estudio, hasta aspectos vinculados a incorporar técnicas de enseñanza del Derecho que incidan en fomentar en el estudiante un mayor interés por la comprensión y análisis del aspecto histórico en el Derecho, tema sobre el cual se centrará el presente trabajo.

La propuesta tiene como punto de partida el interés por desarrollar la competencia de la investigación con apoyo de las Tecnologías de la Información y Comunicación a fin de identificar y aplicar las fuentes del Derecho a la solución de problemas jurídicos, conforme a lo previsto por el indicado Plan de Estudios 2019. A partir de dicha referencia se podrá observar incluso que las Tecnologías de la Información además de ser instrumentos para investigar, también pueden ser instrumentos para favorecer el diálogo interdisciplinario y vehículos alternativos para transmitir el contenido de las investigaciones histórico-jurídicas, susceptible de difundir la cultura jurídica hacia otros sectores de la población. 


\section{EL MATERIAL AUDIOVISUAL COMO HERRAMIENTA AUXILIAR PARA LA} ENSEÑANZA DE LA ASIGNATURA HISTORIA DEL DERECHO MEXICANO

El Plan de estudios 2019 de la Facultad de Derecho, en las competencias generales alude a la capacidad de investigar con apoyo de las Tecnologías de la Información y Comunicación a fin de identificar y aplicar las fuentes del Derecho a problemas jurídicos. Igualmente, dentro de las estrategias didácticas para los procesos de enseñanza-aprendizaje se alude a dichos materiales como parte del análisis de películas. En este marco, se estaría reconociendo que en la labor de investigación y en la estrategia didáctica junto con la información documental, también adquieren una particular importancia las Tecnologías de la Información y Comunicación, para lo cual es significativo indagar los alcances de dichas Tecnologías, en donde el material audiovisual representa una herramienta auxiliar para favorecer el aprendizaje significativo, en donde en todo momento habrá de estar presente la guía del docente para fijar los objetivos de aprendizaje.

En general, término de Tecnologías de Información y Comunicación se concibe como:

Los principios científicos y tecnológicos que permiten desarrollar, potenciar y mejorar el uso y manejo de la información, así como la comunicación de la misma entre distintos sistemas. Tienen como objeto común el proceso de la información en formato digital (Sicilia Urban y García Barriocanal, 2012, p. 12).

Dentro de estas Tecnologías de Información y Comunicación aparecen los audiovisuales, que para Almenara y Barroso (2015) representan vehículos de comunicación didácticos, que junto con transmitir información externa deben ser medios de expresión de las propias ideas y experiencia para los protagonistas del proceso didáctico. Como parte de los medios multimedia, los autores en comento exponen que dichos materiales fomentan la interacción con el usuario, facilitando la navegación y el acceso, así como la individualización del aprendizaje con una finalidad didáctica.

Para Ogable Careaga y González Videgaray (2008, p. 49), "se considera multimedia a la combinación de varios elementos: texto, imagen, sonido, interacción y movimiento, en un solo producto".

En el caso de la asignatura de Historia del Derecho Mexicano, el material audiovisual puede ser de significativa trascendencia para la facilitar la comprensión y profundización de los temas propios de la asignatura, en tanto que puede proporcionar un panorama más amplio del contexto dentro del cual surgieron las normas jurídicas, así como ofrecer un acercamiento a temas particulares de la Historia, a partir de las investigaciones propias de otras áreas. La referencia a dichos audiovisuales involucra aproximar al estudiante a videos documentales, video cápsulas, programas de radio especializados, conversatorios, conferencias, seminarios, coloquios y eventos realizados por instituciones académicas enfocadas en la investigación del área, que de manera enunciativa más no limitativa, apunta a conocer el trabajo realizado por el Instituto de Investigaciones Históricas o el Instituto de Investigaciones Estéticas 
de la Universidad Nacional Autónoma de México, el Colegio de México, el Instituto Nacional de Estudios Históricos de las Revoluciones de México, el Instituto Mora, entre otras instituciones, Asimismo, la referencia a Museos, sitios históricos o arqueológicos puede ser de especial trascendencia para ofrecer información complementaria al estudiante relacionada con los distintos temas de estudio. Las películas igualmente pueden ser un referente interesante bajo el cual se exhorte a los alumnos a realizar cuestionamientos en torno a la precisión o no de la perspectiva del cineasta acerca de un hecho histórico determinado.

Dichos materiales representan instrumentos auxiliares para que el alumnado pueda comprender y profundizar en el estudio de diversos temas, como la importancia política, económica, social y cultural de las virreinas en la Nueva España que puedan aportar datos culturales interesantes para el estudio de la Historia. Adicionalmente, los materiales audiovisuales pueden reforzar la comprensión del contexto histórico bajo los cuales se han emitido decisiones judiciales a lo largo de la Historia del Derecho Mexicano. En este marco, es posible explorar los alcances de la impartición de Justicia en la Nueva España, así como datos en torno a los juicios instaurados en contra de Miguel Hidalgo y Costilla o Maximiliano de Habsburgo. Con ello es posible aproximarnos a la comprensión de temas sociales y culturales presentes en una época histórica determinada, tendientes a enriquecer el horizonte dentro del cual se analizan las normas y principios jurídicos de un determinado periodo.

Adicionalmente, junto con el material documental, los materiales audiovisuales pueden servir para proporcionar un contexto bajo el cual los estudiantes conozcan las posturas antagónicas que han estado presentes en un momento dado dentro de la Historia de México y estén en posibilidades de debatir los distintos temas. A manera de ejemplo, es posible contextualizar las ideas de pueblos indígenas, conquistadores y misioneros, criollos y mestizos, explorar la ideología de los defensores de la Independencia y aquella del ejército realista, el pensamiento de los liberales y conservadores en el siglo XIX, así como los posicionamientos en torno a la regulación de la actividad económica sustentados en el siglo XX en México.

Como se puede apreciar, los materiales audiovisuales están encaminados para ayudar al estudiante a comprender diversos tópicos del temario, así como fomentar la autoevaluación en torno al contenido de los materiales estudiados. Incluso, a través del material audiovisual es posible exhortar a los estudiantes asuman un papel activo en su proceso de enseñanza-aprendizaje, en tanto que pueden identificar medios adicionales para difundir el conocimiento jurídico. 


\section{LA INCORPORACIÓN DE LAS TECNOLOGÍAS DE LA INFORMACIÓN Y COMUNICACIÓN DENTRO DE LAS TÉCNICAS DE ENSEÑANZA DEL DERECHO}

La incorporación de las Tecnologías de la Información y Comunicación como herramientas complementarias en el estudio de la Historia del Derecho Mexicano requieren la adecuada implementación de las técnicas de enseñanza del Derecho para fijar los objetivos bajo los cuales se habrán de utilizar los audiovisuales, así como explorar otros posibles usos de dichos medios. En la perspectiva de Luis Ponce de León Armenta (2003, p. 60):

Las técnicas de enseñanza se sustentan en la actividad de todos los miembros del grupo para la adquisición de los contenidos de enseñanza-aprendizaje, las cuales posibilitan más el intercambio de experiencias y vivencias, el trabajo y la responsabilidad compartida, así como la realización de objetivos de actitudes, hábitos y de integración social.

En este marco, las técnicas de enseñanza del Derecho apuntan a redimensionar las relaciones entre profesor, alumno e institución educativa, que a su vez se reflejen en la práctica e investigación jurídica, y en donde el docente es considerado un medio que intensifica la transmisión de conocimientos, habilidades y destrezas (Ponce de León Armenta, 2004).

Como bien lo indican Esquerre y Huamán (2020, p. 1165):

Las estrategias de aprendizaje deben estar conformadas por la parte informativa, planear la actuación del alumno, monitorear la ejecución y por último evaluar los resultados obtenidos para que ésta funcione como se espera.

En este esquema, la labor del profesor, a través de las técnicas de enseñanza del Derecho, incide en generar confianza en los estudiantes, en desarrollar las estructuras mentales y conocimientos, así como sensibilizar al estudiante con la realidad en la que se encuentra inmerso. Igualmente, se ha de procurar que el alumno asuma un papel activo y construya el conocimiento en su mente a partir de su propia acción e interacción con el objeto de aprendizaje en un proceso paulatino, autónomo y constante, susceptible de transformar su realidad. Bajo este contexto, el adecuado manejo de las técnicas de enseñanza habrá de contribuir a enriquecer de manera sustancial la formación del educando, ya que permiten informar, intercambiar experiencias, propician el surgimiento de habilidades, permiten analizar, evaluar, propician el ejercicio de la reflexión, la creatividad, la iniciativa propia, promueven la renovación y revisión crítica de conocimientos, enseñan a pensar activamente y a escuchar comprensivamente, desarrollan el sentido de cooperación y solidaridad, fomentan las relaciones humanas, se educa para la convivencia.

Enunciados los objetivos de la asignatura Historia del Derecho Mexicano, así como los materiales audiovisuales, procederemos a explorar las posibles ventajas del empleo de los materiales audiovisuales en la enseñanza de dicha asignatura. Si bien de ninguna manera se pretende desplazar a los soportes documentales bajo los que se estudia la Historia del Derecho Mexicano, lo que se busca es identificar una 
herramienta auxiliar de los medios audiovisuales dentro de la enseñanza de dicha asignatura a nivel licenciatura. Aquí, se podría retomar lo que sostiene Selwyn (2016), en el sentido de que la digitalización de la educación apunta a cambios sustanciales y reformas educativas, con lo cual se ha de buscar el potencial de la tecnología digital para sustentar mejores formas de educación.

Aunque se reconoce que en cierta manera todavía existe dificultad para implementar las tecnologías de la información, ya sea por escepticismo en los resultados de su implementación, o por las dificultades que pueden existir por el número de estudiantes y espacio de las aulas convencionales, sin embargo, el interés por procurar la comprensión integral del temario, el diálogo interdisciplinario y la posibilidad de identificar formas alternativas de difundir la investigación histórico-jurídicas, son un aliciente para continuar con la búsqueda de innovaciones para enseñar Derecho.

En todo caso, es importante que el profesor mantenga un criterio razonable al momento de elegir a los materiales audiovisuales dentro de la enseñanza de la Historia del Derecho Mexicano, con la finalidad de obtener el máximo provecho de dichos materiales:

De manera enunciativa, a continuación se exponen algunas sugerencias para el docente para emplear adecuadamente los materiales audiovisuales:

- Realizar la planeación, con el diagnóstico de necesidades y definición de objetivos, tareas y tiempos (Ogable Careaga y González Videgaray, 2008).

- Seleccionar materiales acordes al tema estudiado. En esta selección de materiales es altamente recomendable analizar aspectos como la institución académica que elabora el material, la idoneidad de los contenidos del material audiovisual y determinar los aspectos que pueden fomentar un pensamiento crítico en el estudiante.

- Elaborar un cuestionario que permita que el alumno identifique las principales ideas del material audiovisual.

- Realizar recomendaciones de libros o artículos académicos para que el estudiante pueda profundizar sobre los temas analizados, y en su caso, confrontar con la información contenida en el medio audiovisual.

- Promover portafolios en los que los estudiantes identifiquen líneas de investigación históricojurídicas novedosas que les interesaría desarrollar, y en su caso, valorar cómo se podría comunicar los resultados tanto por medios documentales como por soportes audiovisuales.

Como se puede apreciar, los materiales audiovisuales exhortan a que el docente considere que junto con la actualización en cuanto a los contenidos propios de la disciplina, es relevante contar con conocimientos en torno a las tecnologías de la información y asuma la relevancia de los medios 
audiovisuales en la enseñanza del Derecho, a efecto de generar condiciones idóneas para favorecer el proceso de enseñanza-aprendizaje.

Si apuntamos a los cambios sustanciales y la necesidad de buscar el potencial de la tecnología digital, en los términos que señalaba Selwyn, es posible apuntar para alcanzar dos objetivos a corto plazo y uno a largo plazo que se obtendrían al implementar las referidas Tecnologías en la enseñanza de la Historia del Derecho Mexicano. A corto plazo, el empleo de las Tecnologías de la Información y Comunicación puede favorecer la comprensión de las circunstancias político, económicas, sociales y culturales que incidieron en la conformación del Derecho en una época determinada, la reflexión crítica y sensibilizar al estudiante de la relevancia del diálogo interdisciplinario para contar con una visión integral del fenómeno jurídico. A largo plazo, se puede motivar al estudiante a considerar el empleo de los materiales audiovisuales para exponer los resultados de las investigaciones histórico-jurídicas, con lo cual el estudiante no sólo será un sujeto pasivo receptor de los contenidos de los medios audiovisuales, sino que incluso, se exhorta a que sea un sujeto activo en la creación de nuevo material para exponer sus investigaciones.

Para pronta referencia es conveniente apuntar algunas notas en torno a las implicaciones que tendría el empleo de las Tecnologías de la Información y Comunicación en torno a la reflexión crítica del estudiante con los contenidos de la asignatura, la sensibilización del diálogo interdisciplinario, así como la comunicación y difusión de las investigaciones histórico-jurídicas a través de medios audiovisuales.

a) Comprensión y reflexión crítica del estudiante de los contenidos de la asignatura.- La reflexión crítica de los contenidos de la asignatura apunta a sensibilizar al estudiante de que el Derecho es un fenómeno complejo, en cuya comprensión es significativo que se tengan referentes históricos, políticos, económicos, sociales y culturales para comprender la conformación, sentido y devenir del fenómeno jurídico. Asimismo, conforme a lo adelantado previamente, la aproximación con los documentales y conferencias contenidas en soportes audiovisuales es un referente inicial para que posteriormente el alumno profundice para explorar los alcances que el Derecho tiene en una época histórica determinada.

b) Sensibilización en torno al diálogo interdisciplinario.- Por lo que concierne al diálogo interdisciplinario, se estima que a través de los materiales audiovisuales, los alumnos pueden tener una referencia inicial a la manera en que desde otras disciplinas se aborda un aspecto jurídico, o en su caso, a la metodología, teorías o ideas subyacentes en otras disciplinas. De esta manera, a través de los materiales audiovisuales es posible construir las bases para fomentar un acercamiento con otras disciplinas del saber que favorezcan la comprensión del fenómeno jurídico. En este rubro, ya se vislumbra que el estudiante no sólo debe tener una actitud pasiva frente a los materiales 
audiovisuales que se le han presentado, sino que en cierta medida se generan condiciones para que el alumno pueda construir su conocimiento a partir de un posible diálogo con especialistas de otras disciplinas.

c) Comunicación y difusión de las investigaciones histórico-jurídicas: Conforme a lo mencionado previamente, se puede vincular el uso del material audiovisual con un objetivo a largo plazo, consistente en considerar a los soportes audiovisuales como un canal de comunicación y difusión adicional para exponer los resultados de las investigaciones histórico-jurídicas. Es decir, lo que se busca es que el alumno tenga apertura a formas adicionales de transmitir el conocimiento derivado de su investigación, más allá de la exposición documental para difundir su investigación. Desde luego, se entiende que existen circunstancias en las cuales necesariamente se tendrá que recurrir al soporte documental para transmitir la investigación, sin embargo, existen temas que valdría la pena difundirlos a través de los medios audiovisuales, con la finalidad de que sean del conocimiento no sólo del sector de juristas, sino incluso de la población como tal.

Si bien dentro del aula parecería difícil que durante el semestre se concrete la difusión de una investigación histórico-jurídica, a través de los medios audiovisuales, el docente podría solicitarle a los estudiantes elegir un tema que más les haya parecido interesante, delinear las líneas de investigación e identificar los medios bajo los cuales estimarían conveniente difundir los resultados de la investigación. Aquí se apreciaría la posibilidad de exponer públicamente un trabajo, un proyecto o contenido derivado de la realización de las actividades de las Tecnologías de la Información y Comunicación para el aprendizaje y desarrollo de habilidades de producción personal y difusión pública del conocimiento, en los términos apuntados por Area Moreira y Correa Gorospe (2010).

Con lo anterior, además se favorecer la comprensión de los temas, se daría un impulso adicional a la investigación jurídica, la cual dependerá de la forma y concepto que se tenga del Derecho, de la perspectiva de la ciencia jurídica y de la orientación jurídica metodológica empleada (López, 2002, p. 264). Si bien hasta ahora se ha privilegiado el aspecto normativo del fenómeno jurídico, marginando los aspectos contextuales de la propia norma (Witker y Larios, 2002, p. 241), la aproximación que el estudiante tenga con la Historia, a través de los materiales audiovisuales, le permitirá ofrecer nuevas líneas de investigación e incluso aproximarse a formas alternativas de difundir el conocimiento jurídico y resultados de la investigación jurídica. Aquí es donde ya se fomentaría un rol activo de los estudiantes en relación con los medios audiovisuales, en tanto que se vería a sí mismo como un agente generador de conocimiento y capaz compartir sus aportaciones de investigación con la población en general, de una manera pronta y accesible. 


\section{CONCLUSIONES}

Los materiales audiovisuales en la enseñanza de la Historia del Derecho Mexicano, a nivel Licenciatura, son significativos para ayudarle al alumno a comprender el contexto bajo el cual se ha conformado el Derecho en una época determinada, añadir elementos de autoevaluación del estudiante, fomentar la reflexión crítica, enriquecer el diálogo interdisciplinario, así como explorar distintas formas de divulgación de la investigación jurídica, bajo las cuales los estudiantes puedan exponer los resultados de las investigaciones relacionados con la conformación y elementos formativos del Derecho Mexicano.

En esta propuesta, las técnicas de enseñanza del Derecho adquieren un rol significativo, a partir de las cuales es posible seleccionar los materiales audiovisuales idóneos para explorar determinados temas de la asignatura, motivar la identificación de líneas de investigación histórico-jurídico adicionales, así como fijar los objetivos y actividades de aprendizaje. Así, la educación basada en la exposición del profesor y apoyada en el estudio de fuentes documentales se puede enriquecer con el uso razonable de otros recursos didácticos como la incorporación de las Tecnologías de la Información y Comunicación que complementen la formación en las aulas.

Lo anterior, exhorta a continuar con la reflexión de los retos que tiene la docencia jurídica en el siglo XXI en la formación de juristas críticos y comprometidos con la transformación social. 


\section{REFERENCIAS BIBLIOGRÁFICAS}

Area Moreira, M. y Correa Gorospe, J. M., "Las TIC entran en las escuelas. Nuevos retos educativos, nuevas prácticas docentes", en Juan de Pablo Pons et. al. (Coord.) (2010). Políticas Educativas y Buenas Prácticas con Tic. 2010. Barcelona, España: Editorial Graó.

Esquerre Quispe, José Dolores y Huamán Castro, Milagros Cecilia, “Aplicación de videotutoriales para la mejora de las competencias matemáticas en los estudiantes de pregrado de la Universidad de San Martín de Porres", South Florida Journal of Development, Miami, v.2, n.1, apr/jun 2021, pp. 1161-1170. https://southfloridapublishing.com/ojs/index.php/jdev/article/view/216/211

López Durán, R. (2002). Metodología Jurídica. México: Iure Editores.

Margadant, G. F. (2011). Panorama de la Historia Universal del Derecho. México: Porrúa.

Ogable Careaga, I. y González Videgaray, M. (2008). Nuevas Tecnologías y Educación, diseño, desarrollo, uso y evaluación de materiales didácticos. México: Trillas.

Pérez de los Reyes, M. A. (2008). Historia del Derecho Mexicano, México: Oxford.

Ponce de León Armenta, L. (2003). Docencia y didáctica del Derecho. México: Porrúa e Instituto Internacional del Derecho y del Estado.

(2004). Metodología del Derecho. (6a. ed.), Ciudad de México, México: Porrúa.

Sicilia Urban, M. Á. y García Barriocanal, E. (2012). Aprendizaje y tecnología de la información y la comunicación, Alcalá, España: Centro de Estudios Financieros.

Selwyn, N., (2016). Is Technology good for Education? Cambridge, Reino Unido y Malden, Estados Unidos de América: Polity Press.

Soberanes Fernández, J.L. (2012). Historia del Derecho Mexicano. (15a. ed.), México: Porrúa.

Witker Velázquez, J. y Larios Velasco, R. (2002). Metodología Jurídica. (2a. ed.). México: McGraw-Hill. Recursos electrónicos

Plan y los Programas de Estudio de la Licenciatura en Derecho Modalidad Educativa Presencial (Sistema Escolarizado), (2019), tomo I, recuperado de https://www.derecho.unam.mx/escolares/archivos/tomoI.pdf 